\title{
A Custom Video Retrieval System in Education: Student Perceptions
}

\author{
Peter Tiernan \\ Dublin City University, Ireland
}

\begin{abstract}
In this paper, student perceptions of using a custom video retrieval system as a tool to accomplish an academic task in education were investigated. The study begins by examining the interrelated themes which impact on students use of video in this way, including digital literacy, the learning value of video, and video retrieval systems. The study gathers students' perceptions of the tools available through the video retrieval system to source and share video content for their work. Findings indicate that the search functionality provided a sophisticated means for students to locate content relevant to their work, and that the social features enabled them to share and recommend content to members of their group. The paper also presents a series of conclusions and recommendations for future use of video retrieval systems in education.
\end{abstract}

Keywords: Video retrieval systems, digital literacy, online video, video search, group work

\section{Introduction}

The near ubiquitous availability of digital video [1] and its integration into many aspects of our lives [2] has led to a number of interrelated strands of research concerned with how users source and engage with video content online. In the field of education, digital literacy is seen as an essential skill [3] which is concerned with how students' source, evaluate, communicate with, and integrate digital content into their work [4]. From a technical perspective, much research has been conducted on improving the efficiency of video search through Video Retrieval System (VRS) technology, and the implementation of related features [5]. The purpose of this paper is to examine the implementation of a custom VRS and its evaluation in practice by university students. In order to do so, a number of interrelated themes are examined.

\section{Literature Review}

Digital literacy is increasingly recognised as an essential skill for the modern world [3] and an essential capacity for learning [6]. Initial definitions for digital literacy date back to the late 1990s when Gilster [7] stated it was "the ability to understand and use information in multiple formats from a wide range of sources when it is presented via computers" (p. 1). Even in this early definition, the focus was not only on finding data online, but also on how users evaluate this information for use in daily tasks. Since the 1990s a plethora of research has been carried out on the area of digital literacy, and while the term now incorporates a range of ideas and competencies, there is relative agreement among scholars about its core aspects. Authors have defined digital literacy in terms: of using digital tools to identify, evaluate, analyse, and synthesise digital resources [8]; searching for, evaluating, understanding, and integrating data [9]; using technology to collect, analyse, and evaluate information [4]; construct new information using digital tools [4]; communicate clearly with others using digital tools; finding and evaluating digital content, then using this information to tackle an authentic problem [10].

We understand from earlier studies that video content can contribute effectively to the learning experience [11], especially when used in conjunction with traditional teaching strategies [12]. The multi-modal nature of video has a positive impact on learner motivation and engagement [14] by holding the viewers' attention and interest [13], and can even build an emotional connection between the viewer and the material [15]. The nature of video means that ideas and concepts can be repeated and rewatched [16], if the viewer is unclear. The pace of video can also be changed, either slowed down or sped up, potentially improving understanding [17]. Video can be used to break down complex skills and tasks in a step-by-step manner [18] and introduce ideas, opinions, locations, and moments in time that might otherwise be inaccessible [19]. We know from the existing literature that it is important to consider how video is integrated into the learning experience. Previous work suggests that video content is clearly linked to the learning objectives and that students are encouraged to engage meaningfully with the content through guided questions, reflection, discussion and follow-on activities [20]. It is also recommended that video helps students form links to existing knowledge by adding content which provides examples, expert opinion, and real world contexts [21]. 
VRSs are concerned with improving access to digital video so that users can more easily discover and reuse content [22]. This is achieved by employing two information retrieval processes which categorise and display the content for the user in a meaningful way. When a user searches for content on YouTube, the search is based on the title of the video, the short description, key words and any other metadata that has been manually added. The search does not take into account the actual spoken content of the video [23]. Content-based analysis uses a technique called spoken word analysis to search through the transcript of the video [24] so that users can locate content based on what is being discussed rather than the metadata alone. Next the transcript is linked to the video timeline so that specific points in the video can be access through search. VRSs also improve search functionality through video segmentation. Video segmentation uses a technique called shot boundary detection which actively segments search results into concise, usable units. Shot boundary detection [25] is "the process of automatically detecting the boundaries between shots in a video" (p. 1). These shots then become available to the user as standalone segments, independent of the 'full' video. By combining content-based analysis and video segmentation, VRSs can align search terms to specific segments of video (see Figure 1) text for illustration purposes only).

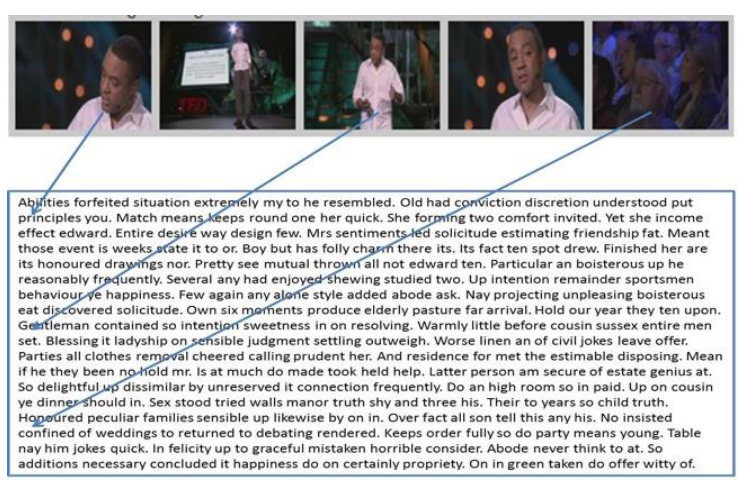

Figure 1 . Content analysis and shot boundary detection

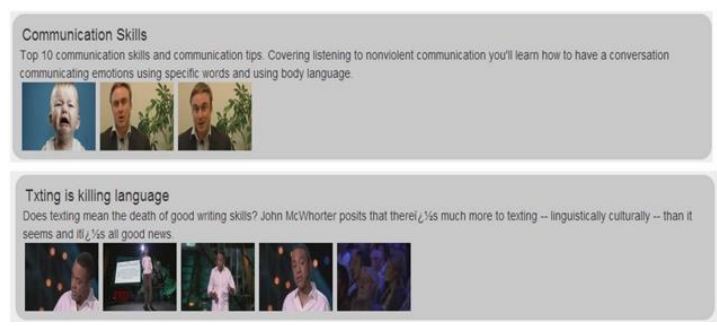

Figure 2. Video search and segmentation in action

For the user, this means that searching for specific words or phrases returns a list of video segments which, when clicked, bring the user to the point in the video where that word or phrase is used (see Figure 2).

Many quantitative studies have been carried out which evaluate the effectiveness of VRSs in terms of search accuracy and response times. Literature on the use of video as a means to increase student engagement is relatively common. Meanwhile, discussions on digital literacy have increased in number, with a particular focus on how students can access and use digital content as part of their academic work. This paper aims to contribute to discussion in these related fields by examining the use of a VRS in practice, whereby students use the system to locate video content and use it in their academic work.

\section{Methodology}

To gather student perceptions of a VRS in education, they were tasked with creating a group presentation, the design of which was to be informed by video content made available through a custom VRS. The system contained search functionality and sharing features designed to support students in their task. Following completion of their work, students were asked to reflect on their experiences using the VRS' features to support the development of their presentations. Further details are now presented for the reader.

\subsection{Sample}

The sample for this study comprised of 140 students of a B.Sc. in Education and Training. Data was gathered from two first year cohorts, containing 70 students in each. All participants were taking part in a module title 'Social and Personal Development with Communication skills' which was a standard timetabled module, delivered two hours per week.

\subsection{The module}

This module was selected for use in this study as it provided ample opportunity to incorporate video content into the student learning experience, and thus investigate the impact of the VRS. The module 'Social and Personal Development with Communication Skills' is a practical module which is designed to help first year students engage fully with the academic learning experience and university life. Topics delivered throughout the 12week semester included: goal setting; time management; learning styles and strengths; creativity and creative thinking; communication skills; conflict management; and stress management. 


\subsection{Description of process}

What makes this study unique is the practical, real world context in which the VRS was deployed and used by participants. This provided an opportunity to gain qualitative insights into the performance of a VRS for a specific task. Participants in this study were given a group assignment in which they were required to work collaboratively to source, share, comment on, integrate and reference video. The assignment comprised of the development of a group presentation on one of the module topics, which are outlined above. Information for their topics was drawn from lectures, readings and other information provided to participants. However, students were instructed to inform the design and delivery of their presentations from the video content which was made available to them on the VRS. The VRS contained a total of 120 videos including University produced video; TED talks; corporate training material; uploaded television content; YouTube channel content. Content on the VRS was reviewed by the researcher to ensure quality and relevance to the participants work.

In addition to the information retrieval features outlined above, the VRS was enhanced with features which enabled users engage with video content for group work. A group system was developed which allowed students to be assigned to groups, within which they could freely share video segments where comments and replies were only visible to members of that specific group (see Figure 3).
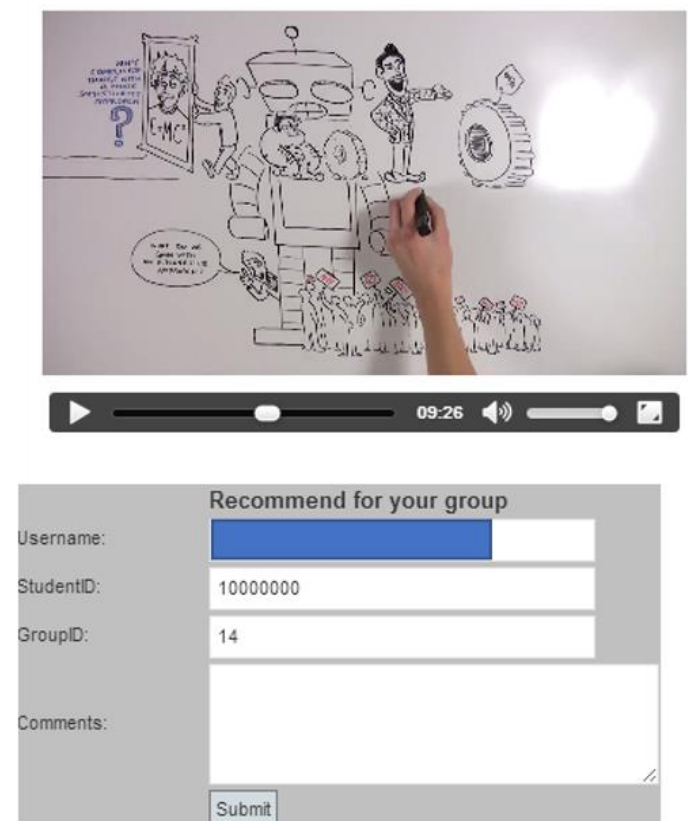

Figure 3. Commenting and sharing

It was envisaged that this would encouraged students to recommend content to each other and fostered a sense of achievement among groups.
Students were given 13 weeks to complete the assignment, providing ample time to engage with the video content and features of the VRS.

\subsection{Instruments}

Data collection comprised of a questionnaire and reflective documents. The questionnaire, which was sent to students after their group assignment was complete, collected qualitative and quantitative data on participants opinions of using the VRS to complete their task. The reflective documents, again submitted after assignments were completed, contained participant reflections on the benefits, challenges and potential improvements that could be made to the VRS for use in a group context.

\subsection{Procedure}

As previously mentioned, questionnaires and reflective documents were submitted following the completion of group assignments. Out of the 140 students, a total of 124 reflective documents and 83 questionnaires were returned, giving a response rate of $89 \%$ and $59 \%$ respectively.

\subsection{Data analysis}

Quantitative data for this study was analysed using simple statistical analysis. Analysis of the qualitative data was conducted using the constant comparative technique [26]. This is an iterative process which analyses the words and phrases used by participants and places them into emerging categories. As categories emerge, rules of inclusion are used to place boundaries on categories and signal when a new category should be created. Techniques based on the work of Guba [27], were used to ensure the viability and credibility of the data categories. Data was checked to ensure consistency within categories, linkages across categories, and that categories were inclusive of the available data. A record was kept of the data analysis process so that it could be reproduced by a competent judge in the future, should this be necessary.

\section{Findings and Discussion}

Findings from this study are now presented in thematic format. These are presented using the data gathered from the questionnaires and reflective documents submitted by participants. These findings, detailed in the following sections, indicate that the search and sharing functionality were successful in improving student engagement with video. Following these findings, overall conclusions are drawn and recommendations made. 


\subsection{The VRS had a positive impact on students' ability to source video content}

Data gathered from students indicated that the VRS had a predominantly positive impact on their ability to locate and use information. $73 \%$ of questionnaire respondents $(n=60)$ rated the VRS 'good' or higher, for helping them to locate relevant content. The qualitative data contained within student reflections, shed light on some of the positive aspects of using the VRS for their task. Their comments $(n=48)$ suggested that the sophisticated search functionality allowed students to locate content relevant to their work. Students commented that P31 - I did not have to trawl through endless footage of unnecessary video to find what I wanted', that the VRS 'P01 - has an easier search than other video websites because it searches for the key word you looked for in the search box'. This helped to provide a more accurate search, or as one student said it helped them to find 'P20 footage of exactly what I was looking for'. Students also highlighted the video segmentation feature as a useful tool in refining their search and presenting them with concise information. Comments $(n=32)$ included 'P85 - it avoided going through extremely long videos' by being brought 'P109 - to the exact point in the video', and that the 'P22 - method of lessening the videos down to the specific ones you need for your assignment is a brilliant way to do your research and get the exact information you need'.

While the many of comments were encouraging, some students did experience difficulty locating content on the VRS. The main area of concern appeared to be the lack of a clear relationship between what the student typed in the search box, and the list of segments that was presented as results. These comments $(n=12)$ included assertions that they 'P16 - found the system to be quite vague', saying that it was 'P107 - sometimes hard to find a video with reference to what I was searching for'. Included in their concerns were some suggestions for improvement - ideas that might make the relationship between search term and results list clearer. Key among these was the inclusion of text summaries for video segments. Students said 'P03 if it showed the first sentence of that segment so that we know exactly what that part is talking about' and 'P29 - descriptions for each segment saying what this segment is about.'

Data here suggests that the VRS was successful in enabling students to locate video for their assignment. From a digital literacy perspective, it appears to have aided them in navigating through non-textual information while remaining focused on their task [28]. Feedback from students suggests it may be possible to improve the representation of search results by presenting them to students in a more meaningful way, perhaps through the inclusion of text summaries for individual video segments.

\subsection{Student impressions of video sharing and commenting for a group assignment}

The video sharing and commenting features which were implemented on the VRS also seem to have had a positive impact on how students used video in their group work, especially on the ways in which they communicated with each other during the search process [4]. The tools were particularly useful in enabling groups to gather more content than would have been possible if students were working alone. Their comments $(n=112)$ included: 'P43 - sharing was a bonus as I was able to view videos that other group members suggested that I might not have found myself' and 'P02 - each member could recommend videos they felt were effective'. The ability to share their selection also appears to have improved decision making, with students saying it helped them to 'P67 communicate with other members in my group' and to 'P19 - make a more informed decision' on what to view. The ability to add comments alongside video shares, allowed students to share ideas and express opinions [10] related to their assignment. Comments from students suggested that they were able to 'P79 - elaborate in their own words on the reasons they chose and tagged the video' and explain why they 'P54 - found that specific video useful'. The feature worked two ways in that it also allowed them to 'P41 - get different views and opinions' on videos what were shared by other members of the group. Comments $(n=22)$ suggested that students began using the sharing and commenting features to delegate tasks by 'P06 - could dividing up search terms', meaning they could work as a team to 'P33 - work together to find a variety of videos'.

The comments presented above are supported by the actual interactions $(n=92)$ that occurred between the group members on the system, which comprised of: 1) Sharing video segments with the groups, and 2) Sharing opinions and views on video segments. The majority of interactions $(n=56)$ fell into the first category, however there was some nuance in how these were shared. Some students used the sharing feature to highlight the relevance of a video segment to the group (see Figure 4), with comments like 'P31 - This is very good to help with the narration and presentation' and 'P61 - This video showed me ways of giving a good speech for a presentation'. Others $(n=28)$ opted to attach a personal message to encourage other to view the video, such as 'P65 - I recommend watching', and 'P82 - I think this video could help us a lot'. 


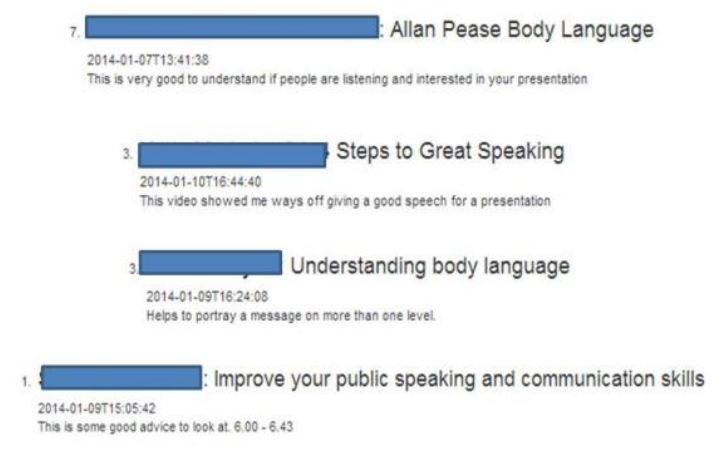

Figure 4. Segment share with comment

Sharing of opinions and views $(n=40)$ included examples (see Figure 5): 'P30 - I found this video to be a good recommendation because from looking at the videos on body language I tried to take this action into account by having open palms and to not be controlling the conversation and to be more welcoming about other people's opinions on what we should do' and 'P81 - Here's another one by the same guy - it's interesting because it's about persuasion which is an important part of the communication process'. These interactions clearly demonstrate the use of the VRS to share opinions and viewpoints around digital video, in particular the value of these tools when video is incorporated into an authentic task [20] for students to complete.

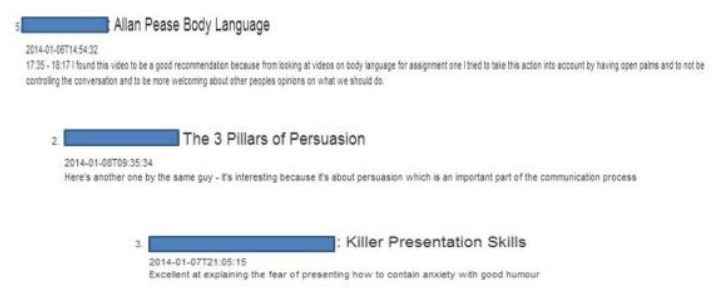

Figure 5. Segment share with opinions

Data presented in this section suggests that the video sharing and commenting features of the VRS had an overwhelmingly positive impact on students ability to use digital video for their group assignment. The features facilitated group work by allowing members of the group to recommend content, meaning more video could be viewed by the group than may have been sourced along. The features also facilitated the sharing of views and opinions which allowed members to share reasons for their choices and encouraged other members to view specific sections of video. Viewing this data through a digital literacy lens shows students using digital tools to communicate around video [30][4] in range of ways, such as the ability to share relevant content [28] and communicate relevant information which supports the completion of their task [10].

\section{Conclusion}

Findings indicate that the VRS that was employed in this study received a positive response from students, who used it to complete an authentic academic task. The advanced search functionality, which was powered by content-based analysis and video segmentation, provided a sophisticated means for students to locate content relevant to their work. While students appreciated the precise nature of the search and the concise segments provided, some areas of improvement were identified. Key among these where students concerns around the lack of a clear relationship between their search term and the resulting list of video segments. Their own comments suggested the inclusion of automated text summaries, so that search terms could more easily be spotted within individual video segments. The development and implementation of the video segment sharing and commenting features appears to have been very successful. Students were easily able to use the features to share and recommend content to members of the group, with the addition of comments and opinions allowing them to add reasons for choosing a specific video segment, or further context to their selection. This evidence came through in students own reflections and on the interactions which took place on the VRS.

\section{Recommendations}

The search functionality of the VRS shows great potential in enabling students to source video content for their work. Students responded well to an authentic task on the area of communication skills, when provided with themes and categories to guide their search. Future work could investigate the use of a VRS across other subject disciplines and also examine the presentation of search results through text summaries or other means. Allowing students to operate in pre-defined, closed groups, enabled them to share content and work together effectively to locate and incorporate video. Future studies might investigate how students work in groups to locate content which comes from more disparate video sources.

\section{Limitations}

The sample for this study was 140 undergraduate education students. While this represents a significant sample for qualitative study, larger scale studies may be needed if claims are to be made about the effectiveness of VRS features more broadly. Data drawn from a more diverse subject and student cohort may yield different results and experiences. This research used video content which was hosted on a closed platform, with content only updatable by the researcher. Further studies could broaden the 
scope and examine the effectiveness of the VRS search and segmentation features when linked with a range of online video repositories. The sharing and commenting features were designed to facilitate group work, future studies may implement alternative features which could yield different results. Finally, qualitative academic literature on how students evaluate VRSs for use with academic work is uncommon. This means that as research in the area progresses, new alternative themes for study may emerge which were not addressed in this paper

\section{References}

[1] Anderson, M., (2015). "5 Facts About Online Video, For YouTube's 10th Birthday", Pew Research Centre. Available from: http://www.pewresearch.org/facttank/2015/02/12/5-facts-about-online-video-for-youtubes -10th-birthday/. (Access Date: 30 March 2021).

[2] Riismandel, P., (2016). "The State of Education Video 2016”, Available from: http://www.streamingmedia.com/ Articles/Editorial/Featured-Articles/The-State-of-Educati -on-Video-2016-110211. Aspx. (Access Date: 30 March 2021).

[3] Pérez-Escoda, A., García-Ruiz, R., and Aguaded, I., (2019). "Dimensions of digital literacy based on five models of development/Dimensiones de la alfabetización digital a partir de cinco modelos de Desarrollo", Educación, 31(2), pp. 232-266.

[4] Kim, K.T., (2019). "The structural relationship among digital literacy, learning strategies, and core competencies among south korean college students", Educational Sciences: Theory and Practice, 19(2), pp. 3-21.

[5] Schoeffmann, K. Bailer, W. Gurrin, C. Awad, G. and Lokoc, J., (2018). "Interactive Video Search: Where is the User in the Age of Deep Learning?", In Proceedings of the 26th ACM international conference on Multimedia, , pp. 2101-2103.

[6] Rohatgi, A., Scherer, R., and Hatlevik, O.E., (2016). "The role of ICT self-efficacy for students' ICT use and their achievement in a computer and information literacy test", Computers and Education(102), pp. 103-116.

[7] Gilster, P., (1997). "Digital Literacy", Wiley Computer Publications.

[8] Martin, A., (2005). "DigEuLit-a European framework for digital literacy: a progress report", Journal of eLiteracy, 2(2), pp. 130-136.

[9] Ng, W., (2012). "Empowering scientific literacy through digital literacy and multiliteracies", Nova Science Publishers.

[10] Churchill, N., (2020). "Development of students' digital literacy skills through digital storytelling with mobile devices", Educational Media International, 57(3), pp. 271-284.
[11] Nagy, T.J., (2018). "Evaluation of online video usage and learning satisfaction: An extension of the technology acceptance model", The International Review of Research in Open and Distributed Learning, 19(1),

[12] Bohloki, M., Makatjane, T.J., George, M.J., and Mokuku, T., (2019). "Assessing the Effectiveness of using YouTube Videos in Teaching the Chemistry of Group I and VII Elements in a High School in Lesotho", African Journal of Research in Mathematics, Science and Technology Education, 23(1), pp. 75-85.

[13] Meseguer-Martinez, A., Ros-Galvez, A., RosaGarcia, A., (2017). "Satisfaction with online teaching videos: A quantitative approach", Innovations in Education and Teaching International, 54(1), pp. 62-67.

[14] Bower, M., (2017). "Design of technology-enhanced learning; integrating theory and practice", Bingley: Emerald Publishing.

[15] Sweeney, K., and Baker, P., (2018). "Promoting empathy using video-based teaching", Clinical Teacher, 15(4), pp. 336-340.

[16] Weeks, B.K. and Horan, S.A. (2013). "A video-based learning activity is effective for preparing physiotherapy students for practical examinations", Physiother, 99(4), pp. 292-297.

[17] Kettle, M., (2020). "How videos are used in secondary school physics teaching", Physics Education, $55(3)$.

[18] Srinivasa, K., Chen, Y., and Henning, M.A., (2020). "The role of online videos in teaching procedural skills to post-graduate medical learners: A systematic narrative review”, Medical Teacher, 42(6), pp. 689-697.

[19] Snelson, C. (2008). "Web-based video in education: Possibilities and pitfalls", In Proceedings of the Technology, Collages and Community Worldwide Online Conference, pp. 214-221.

[20] Moskovich, Y., Shark, S., (2012) "Using films as a tool for active learning in teaching Sociology", The Journal of Effective Teaching, 12(1), pp. 53-63.

[21] Mitra, B., Lewin-Jones, J., Barrett, H., and Williamson, S., (2010). "The use of video to enable deep learning", Research in Post-Compulsory Education, 15(4), pp. 405-414.

[22] Laughton, P.A., (2012). "Open Archival Information System (OAIS) as a data curation standard in the World Data Centre (Doctoral dissertation)".

[23] Gurrin, C., (2009). "Content-based video retrieval", Encyclopaedia of database systems, USA: Springer, pp. 466-473.

[24] Lew, S.M., Sebe, N., Djeraba, C., and Jain, R., (2006). "Content-based multimedia information retrieval: State of the art and challenges", ACM Transactions on Multimedia Computing, Communications, and Applications (TOMCCAP), 2(1), pp. 1-19. 
[25] Smeaton, A.F., Over, P., and Doherty, A.R., (2010). "Video shot boundary detection: Seven years of TRECVid activity", Computer Vision and Image Understanding, 114(4), pp. 411-418.

[26] Maykut, P., and Morehouse, R., (1994). "Beginning Qualitative Research: A philosophical and practical guide", London: RoutledgeFalmer.

[27] Guba, E.G., (1978). "Toward a Methodology of Naturalistic Inquiry in Educational Evaluation", CSE Monograph Series in Evaluation, 8, pp. 56-57.

[28] Eshet-Alkalai, Y., and Chajut, E., (2009). "Changes over time in digital literacy", CyberPsychology and Behavior, 12(6), pp. 713-715.

[29] Berkhof, M. van Rijssen, H.J. Schellart, A.J. Anema, J.R. and van der Beek, A.J. (2011). "Effective training strategies for teaching communication skills to physicians: an overview of systematic reviews", Patient education and counseling, 84(2), pp. 152-162.

[30] Hague, C., and Payton, S., (2011). "Digital literacy across the curriculum", Curriculum Leadership, 9(10). 